Revista Thema

v.20 Especial 2021

p. $73-88$
DOI: http://dx.doi.org/10.15536/thema.V20.Especial.2021.73-88.1889

ISSN: 2177-2894 (online)
CIÊNCIAS DA SAÚDE

\section{Dengue e COVID-19 em Minas Gerais:} análise macrorregional dos casos, internações e investimentos na assistência à saúde durante a pandemia

Dengue and COVID-19 in Minas Gerais: macro-regional analysis of cases, hospitalizations and investments in health care during the pandemic

Camila Gouvêa Facure ${ }^{1}$, Claiton Luiz de Almeida Filho², Milena Vieira Dias do Santos ${ }^{3}$, Thaís Borba Carneiro ${ }^{4}$,

Stefan Vilges de Oliveira ${ }^{5}$

\title{
RESUMO
}

Este estudo objetiva realizar uma análise macrorregional dos casos, das internações, da infraestrutura de saúde e dos investimentos voltados à dengue e à COVID-19 no estado de Minas Gerais. Trata-se de estudo epidemiológico, descritivo e quantitativo pelo Sistema de Informação Hospitalar (SIH-SUS), Sistema de Informação de Agravos de Notificação (SINAN) e boletins epidemiológicos da COVID-19 da Secretaria de Estado de Saúde de Minas Gerais e do Ministério da Saúde entre janeiro de 2017 a maio de 2020. Registraram 845.352 casos de dengue com 15.366 internações, onerando $\mathrm{R} \$ 6.247 .580 .00$ às 14 macrorregiões do estado. O pico de incidência da dengue esteve entre janeiro a maio dos anos analisados. Para a COVID-19, nota-se aumento dos casos ao longo de 2020 gastando $\mathrm{R} \$ 165.879 .596 .00$ até o presente estudo. Destaque para a macrorregião Centro com maior número de casos por ambas doenças. A dengue, por si só, já prejudica a assistência hospitalar fornecida pelo SUS em Minas Gerais. Com a concomitância temporal da COVID-19 em 2020, o dispêndio ao sistema de saúde agravar-se-á, uma vez que ambas poderão sobrecarregá-lo. Portanto, deve-se focar na atenção primária para otimizar gastos.

Palavras-chave: Dengue; COVID-19; atenção primária à saúde; vigilância em saúde.

\footnotetext{
${ }^{1}$ Discente do curso de Medicina, Universidade Federal de Uberlândia - UFU, Uberlândia/MG - Brasil. E-mail: camila.g.facure@gmail.com

${ }^{2}$ Idem. E-mail: claitonlaf@gmail.com

${ }^{3}$ Idem. E-mail: milenavds15@gmail.com

${ }^{4}$ Idem. E-mail: thais.borbac@gmail.com

${ }^{5}$ Docente do Departamento de Saúde Coletiva da Faculdade de Medicina, Universidade Federal de Uberlândia- UFU, Uberlândia/MG - Brasil. E-mail: stefanbio@yahoo.com.br
} 


\section{ABSTRACT}

This study aims to perform a macro-regional analysis of cases, hospitalizations, health infrastructure and investments aimed at Dengue and COVID -19 in Minas Gerais State. This is na epidemiological, descriptive and quantitative study using Sistema de Informação Hospitalar (SIH-SUS), Sistema de Informação de Agravos de Notificação (SINAN) and epidemiological bulletins of COVID-19 from the Secretaria de Estado de Saúde de Minas Gerais and the Ministério da Saúde between January 2017 and May 2020. It was registered 845.352 dengue cases with 15.366 hospitalizations, costing $R \$ 6,247,580.00$ to the 14 macro-regions of the state. The peak incidence of dengue was between January and May of the years analyzed. For COVID-19 there is an increase in cases over 2020, spending $R \$ 165,879,596.00$ until the present study. Highlight for the "Centro" macro-region with the highest number of cases due to both diseases. Dengue, by itself, already harms the hospital care provided by SUS in Minas Gerais. With the temporal concomitance of COVID-19 in 2020, the expenditure on the health system will aggravate, once both may overload it. Therefore, one should focus on primary care to optimize spending.

Keywords: Dengue; COVID-19; primary health care; health surveillance.

\section{INTRODUÇÃO}

A dengue configura-se como um histórico problema de saúde pública no Brasil e exige um grau de complexidade das ações integradas e intersetoriais de saúde, organizadas em prol do seu controle. Trata-se de uma arbovirose urbana, sistêmica, dinâmica, de amplo espectro clínico e transmitida principalmente pela picada do mosquito Aedes aegypti. (TEIXEIRA; BARRETO; GUERRA, 1999).

Paralelamente às epidemias de dengue, descritas por Teixeira, Barreto e Guerra (1999), desde 1986 no Brasil, tem-se no início do ano de 2020 no país, a doença causada pela infecção do novo coronavírus da síndrome respiratória aguda grave 2 (SARS-CoV-2), nomeada por COVID-19 e decretada como pandemia no dia 11 de março pela Organização Mundial da Saúde (2020).

Tal definição abordada pelo Ministério da Saúde (2020) deve-se em razão de seu alto poder de disseminação mundial, uma vez que a transmissão ocorre de pessoa a pessoa pelo ar, por meio de gotículas expelidas pelo doente durante a tosse, fala ou espirro. Além do contato com objetos contaminados por pessoas doentes e posterior contato com a boca, nariz ou olhos.

Embora essas enfermidades sejam transmitidas de maneira distintas, ambas compartilham aspectos sintomatológicos, tal como a possível presença de manifestação cutânea que é característica da dengue, mas já foi relatada em casos da COVID-19 (YAN et al., 2020), o que pode acometer falsos diagnósticos ou até mesmo coinfecção em meio a coincidência temporal, visto que a epidemia de dengue possui caráter sazonal que conflui com a presença do coronavírus no Brasil. (LORENZ; AZEVEDO; CHIARAVALLOTI-NETO, 2020). 


\section{Edição Especial COVID-19}

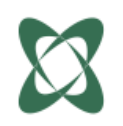

Ademais, as duas doenças manifestam-se com variado quadro clínico de assintomáticas para sintomáticas e, quando possuem sintomas, esses variam de leves até graves, podendo evoluir a óbito, fatores esses desencadeantes, muitas vezes, de imprevisibilidade do diagnóstico, dificultando assim a atuação da atenção primária em saúde e a adoção de manejo clínico adequado. Dessa maneira, tem-se a utilização de serviços hospitalares, como a internação em casos mais graves. (TEIXEIRA; BARRETO; GUERRA, 1999) (MINISTÉRIO DA SAÚDE, 2020).

Além do impacto clínico negativo, o fardo econômico ao Sistema Único de Saúde (SUS) associado a essas doenças tem sido preocupante. No estado de Minas Gerais, A Secretaria de Estado de Saúde (2020) publicou no dia 4 de março de 2020 repasses financeiros complementares, na ordem de $\mathrm{R} \$ 640$ mil para apoio de 29 municípios com alta incidência de casos de dengue. Já em relação ao repasse para a COVID-19, na mesma região, o valor foi de aproximadamente $\mathrm{R} \$ 61$ milhões, publicado em 27 de março de 2020, destinado às ações de saúde para o enfrentamento do novo coronavírus.

Em meio a esse complexo cenário epidemiológico, vale ressaltar que as discrepâncias sociais e as diferenças econômicas inter-regionais no estado de Minas Gerais refletem uma desigual distribuição tanto dos serviços de saúde da rede hospitalar quanto dos complexos sistemas com aparato tecnológico, influenciando nos cuidados para a manutenção da saúde. (OLIVEIRA; SIMÕES; ANDRADE, 2008).

Em consonância com essa situação, evidencia-se então a complexidade da determinação multidimensional da dengue, que define a questão socioeconômica aliada aos fatores climáticos fundamentais para análise das regiões endêmicas, segundo a classificação dos macros e micro determinantes de saúde. (BUSS; PELLEGRINI FILHO, 2007).

Portanto, a dengue, por si só, demonstra ser uma doença recorrente capaz de reduzir a qualidade de vida e demandar a busca pela assistência médica ao longo dos anos no país, principalmente quando ocorre complicações do quadro clínico, situação que agravar-se-á perante o atual contexto de pandemia da COVID-19, na medida em que ambas enfermidades oneram o SUS. Assim, tem-se a necessidade de reformular estratégias de saúde.

À luz do que foi abordado e tendo em vista que o estado de Minas Gerais apresenta discrepâncias socioeconômicas e variantes climáticas, fatores esses determinantes para a distribuição da dengue e da COVID-19, este estudo tem como objetivo realizar uma análise macrorregional dos casos, das internações, da infraestrutura de saúde e dos investimentos empregados para a assistência a dengue e a COVID-19 no contexto pandêmico no estado de Minas Gerais.

\section{MÉTODO}

Realizou-se um estudo epidemiológico descritivo, quantitativo, baseado em dados secundários das doenças dengue e COVID-19, a fim de analisar o impacto de ambas 
as doenças no SUS, especificamente no estado de Minas Gerais, dividido nas suas 14 macrorregiões de saúde definidas no Plano Diretor de Regionalização (PDR) de Minas Gerais, em 2019, sendo as macrorregiões: Sul, Centro Sul, Centro, Jequitinhonha, Oeste, Leste, Sudeste, Norte, Noroeste, Leste do Sul, Nordeste, Triângulo do Sul, Triângulo do Norte e Vale do Aço.

O estado de Minas Gerais, localizado na região sudeste do Brasil, conta com 853 municípios (IBGE, 2018) e possui área territorial de $586.521,123 \mathrm{~km}^{2}$. (IBGE, 2018). De acordo com o censo 2010 do IBGE, sua densidade demográfica é de 33,41 hab./km2 e a população estimada é de 21.168.791, sendo o segundo estado mais populoso do Brasil, de acordo com os dados do IBGE 2019. Conforme o Censo Demográfico de 2010 do IBGE, o Índice de Desenvolvimento Humano (IDH) da região é de 0,73115.

Os dados da dengue foram extraídos do Sistema de Informação Hospitalar do Sistema Único de Saúde (SIH/SUS) e do Sistema de Informação de Agravos de Notificação (SINAN), ambos disponibilizados pelo Departamento de Informática do Sistema do Sistema Único de Saúde (DATASUS) do Ministério da Saúde (BR) e acessados pelo Tabulador de Informações de Saúde (TABNET). Estes dados foram registrados pela Secretaria de Estado de Saúde de Minas Gerais, no período de janeiro de 2017 a abril de 2020 e acessado no dia 10 de junho de 2020.

A análise da dengue considerou a Morbidade Hospitalar do SUS (SIH/SUS) Geral, por local de internação, no estado de Minas Gerais, selecionando: lista de Morbidade CID10 - dengue (dengue clássico) e febre hemorrágica devido ao vírus da dengue com análise em todas as macrorregiões de saúde. Como variáveis, escolheu-se: internações, valor total e médio das internações, valor dos serviços hospitalares e profissionais, média de dias da internação, evolução clínica (óbitos), dias de permanência e taxa de mortalidade. Pelo SINAN foram acessadas informações sobre "Local de residência, mês da notificação, macrorregião de saúde de residência e mês do óbito". Para gerar os dados, usou-se como comando de linha a "Macrorregião de Saúde de Residência", e como coluna "Mês da Notificação" para o período da análise.

Os dados da COVID-19 foram obtidos nos Boletins Epidemiológicos de 2020 disponibilizados pela Secretaria de Estado de Saúde de Minas Gerais e do Painel Coronavírus da Secretaria de Estado de Saúde de Minas Gerais, acessado nos dia 10 e 12 de junho de 2020, e do Portal de Transparência do Estado de Minas Gerais, acessado no dia 13 de junho de 2020. Foram acessados destes o número de casos da COVID-19 para o mesmo recorte espaço temporal acima descrito.

A partir de tais documentos, selecionaram-se dados referentes aos casos confirmados, internações, taxa de ocupação de leitos de UTI adulto e leitos clínicos, pela COVID-19 e outras causas, quantidade de leitos clínicos e de UTI adulto existentes, taxa de mortalidade, população total e óbitos pela COVID-19 no estado de Minas Gerais e em suas 14 macrorregiões. Também foram observadas nesses documentos as estimativas para o ano de 2020 das internações em leitos de UTI, casos e óbitos pela COVID-19.

Após o recolhimento dos dados, foi elaborado gráficos a partir do programa Microsoft Office Excel, versão 2007, analisando as duas doenças no estado de Minas Gerais e 
em suas macrorregiões. Com o objetivo determinar os impactos que a dengue concomitante com a COVID-19 provoca no SUS, apontando quais as macrorregiões de Minas Gerais demandam maiores esforços por parte da rede pública de saúde.

Para realização da presente pesquisa, não foi necessária a emissão de parecer de um Comitê de Ética em Pesquisa (CEP), pois os dados analisados são dados secundários do Ministério da Saúde, não nominal, a qual todos os cidadãos brasileiros têm acesso pela Resolução do Conselho Nacional de Saúde nํ466, de 12 de dezembro de 2012.

\section{RESULTADOS}

No período estudado, no estado de Minas Gerais, ocorreram 15.366 internações por dengue (dengue clássica) e febre hemorrágica devido ao vírus da dengue. O Centro foi a macrorregião com maior número de internações, contando com 4.838 internações $(31,48 \%)$, seguida pelo Triângulo do Norte $(12,87 \%)$, Oeste $(9,58 \%)$ e pelo Sudeste $(8,44 \%)$.

Em 2017, ocorreram 1.735 internações, sendo as macrorregiões Leste, Nordeste e Centro as que apresentaram o maior número de hospitalizações. Em 2018, tem-se uma queda de $27 \%$ no número de internações, 475 internações a menos quando comparada com o ano de 2017. No ano de 2018, foram contabilizadas 1260 internações segundo o SIH-SUS, sendo que 48\% delas ficaram concentradas nas seguintes Macrorregiões: Sudeste, Oeste e Centro. Em 2019, o estado de Minas Gerais presenciou um cenário epidêmico, com um aumento expressivo no número de internações, 10677 no total, um aumento de $747 \%$ se comparado com o ano de 2018.

Desse total, as macrorregiões com o maior número de internações foram, respectivamente, o Centro, Triângulo do Norte e Norte, contabilizando juntas 64,88\%. No período analisado de janeiro a abril de 2020, o SIH registrou 1.694 internações, sendo as regiões do Oeste, Nordeste e Centro, nesta sequência, as regiões com maior número de internações registradas, com $49,46 \%$ do total de internações do estado de Minas Gerais.

Em relação ao custo o valor total das internações em 2017, Minas Gerais teve um gasto de $\mathrm{R} \$ 622.666$ e um valor médio de internação de $\mathrm{R} \$ 358$. No ano de 2018, foram R\$507.032 e um valor médio de $R \$ 402$. No ano de 2019, foram gastos $R \$$ 4.437.742,00 e um valor médio de R\$ 415 por internação. De janeiro a abril de 2020 já foram gastos $\mathrm{R} \$ 680.131$, um valor superior quando comparado ao ano de 2017 e 2018.

Em relação ao tempo e ao custo médio das internações de janeiro de 2017 a abril de 2020, respectivamente, a média de permanência no estado de Minas Gerais foi de 3,3 dias com um custo médio das internações de $\mathrm{R} \$ 406,58$. A macrorregião com o valor médio mais elevado foi a do Vale do Aço, com $R \$ 501,56$, seguido pelo Noroeste ( $R \$$ $499,02)$ e o Oeste $(R \$ 474,79)$. Esses custos médios podem variar em virtude dos valores dos serviços hospitalares e profissionais de cada macrorregião. Em números 
totais, foram gastos $\mathrm{R} \$ 6.247 .574,13$ com as 14 macrorregiões de saúde do estado que totalizaram 50.089 dias permanência no período analisado por esse estudo.

Analisando os óbitos, foram registrados 158 óbitos por dengue (dengue clássica) e febre hemorrágica devido ao vírus da dengue. A macrorregião Centro foi a que mais notificou vítimas fatais, 35 mortes, seguida pelo Oeste Mineiro, com 23, Noroeste, com 18 e Sudeste com 17 mortes registradas. Ademais, a taxa de mortalidade do estado de janeiro 2017 a abril de 2020 ficou em 1,03. Nessa análise, o Noroeste ficou com a maior taxa, acima de todas as macrorregiões com 3,06, seguida por Jequitinhonha, com 1,90, Vale do Aço, com 1,81 e Oeste, com 1,56. Em contrapartida, Leste do Sul, com 0,42, Leste, com 0,62 e Centro, com 0,72, registram as menores taxas.

A tabela 1 mostra os óbitos e as internações por dengue que ocorreram nas 14 macrorregiões de Minas Gerais no período de janeiro de 2017 a abril de 2020. Abaixo segue também as figuras 1 e 2, sendo que a primeira com os dados do SINAN traz um descritivo das doenças dengue nos anos de 2017, 2018, 2019 e 2020 com a COVID-19, no período de janeiro a maio, e a segunda faz uma análise temporal da dengue de 2017 a 2020.

Tabela 1 - Número de casos, óbitos e internações por dengue nas macrorregiões de Minas Gerais, no período de janeiro de 2017 a abril de 2020.

\begin{tabular}{llll}
\hline Macrorregião & $\mathbf{N}^{\circ}$ de casos & Óbitos & Internações \\
\hline Centro & 422.616 & 266 & 4.838 \\
Sudeste & 33.544 & 78 & 1.298 \\
Triângulo do Norte & 73.162 & 70 & 1.978 \\
Sul & 37.426 & 45 & 631 \\
Vale do Aço & 17.761 & 21 & 276 \\
Oeste & 54.496 & 86 & 1.473 \\
Noroeste & 45.650 & 51 & 588 \\
Nordeste & 16.028 & 34 & 1.004 \\
Centro Sul & 5.818 & 4 & 110 \\
Leste & 13.444 & 24 & 970 \\
Triângulo do Sul & 41.911 & 71 & 515 \\
Norte & 52.457 & 27 & 1.239 \\
Leste do Sul & 7.483 & 24 & 236 \\
Jequitinhonha & 6.535 & 3 & 210 \\
Total & $\mathbf{8 2 8 . 3 3 1}$ & $\mathbf{8 0 4}$ & $\mathbf{1 5 . 3 6 6}$ \\
\hline
\end{tabular}

Fonte: Elaborada pelos autores.

Já com relação a COVID-19, o primeiro caso em Minas Gerais foi registrado na 10a semana epidemiológica de 2020 e até o dia 2 de junho foram registrados 13.034 casos e 322 óbitos, com taxa de letalidade de 2,48\%. No entanto, de acordo com o Boletim 
Epidemiológico e Assistencial COVID-19 (edição especial) número 7 da SES-MG, até dia 09 de junho de 2020 já haviam 16.102 casos de COVID-19 no estado, com taxa de letalidade de 2,17\%, 2.567 pessoas necessitaram de internação hospitalar tanto na rede pública quanto na privada em Minas Gerais. A macrorregião Centro registrou a maior frequência de COVID-19, com 4.663 casos e 108 óbitos, seguido do Sudeste, com 1.508 casos e 60 óbitos, e o Triângulo do Norte, com 1.268 casos e 31 óbitos. Juntas, essas macrorregiões somam $57,1 \%$ de todos os casos e $52,5 \%$ da população do estado. Das 14 macrorregiões existentes em Minas Gerais, 7 delas concentram $80,4 \%$ dos casos: Centro (35,80\%), Sudeste (11,60\%), Triângulo do Norte $(9,70 \%)$, Sul $(9,30 \%)$, Vale do Aço $(5,50 \%)$, Oeste $(4,40 \%)$ e Noroeste $(4,10 \%)$.

Figura 1 - Casos de dengue nos anos de 2017, 2018, 2019 e 2020 e da COVID-19 no ano de 2020, no período de janeiro a maio de acordo com o SINAN-MG.

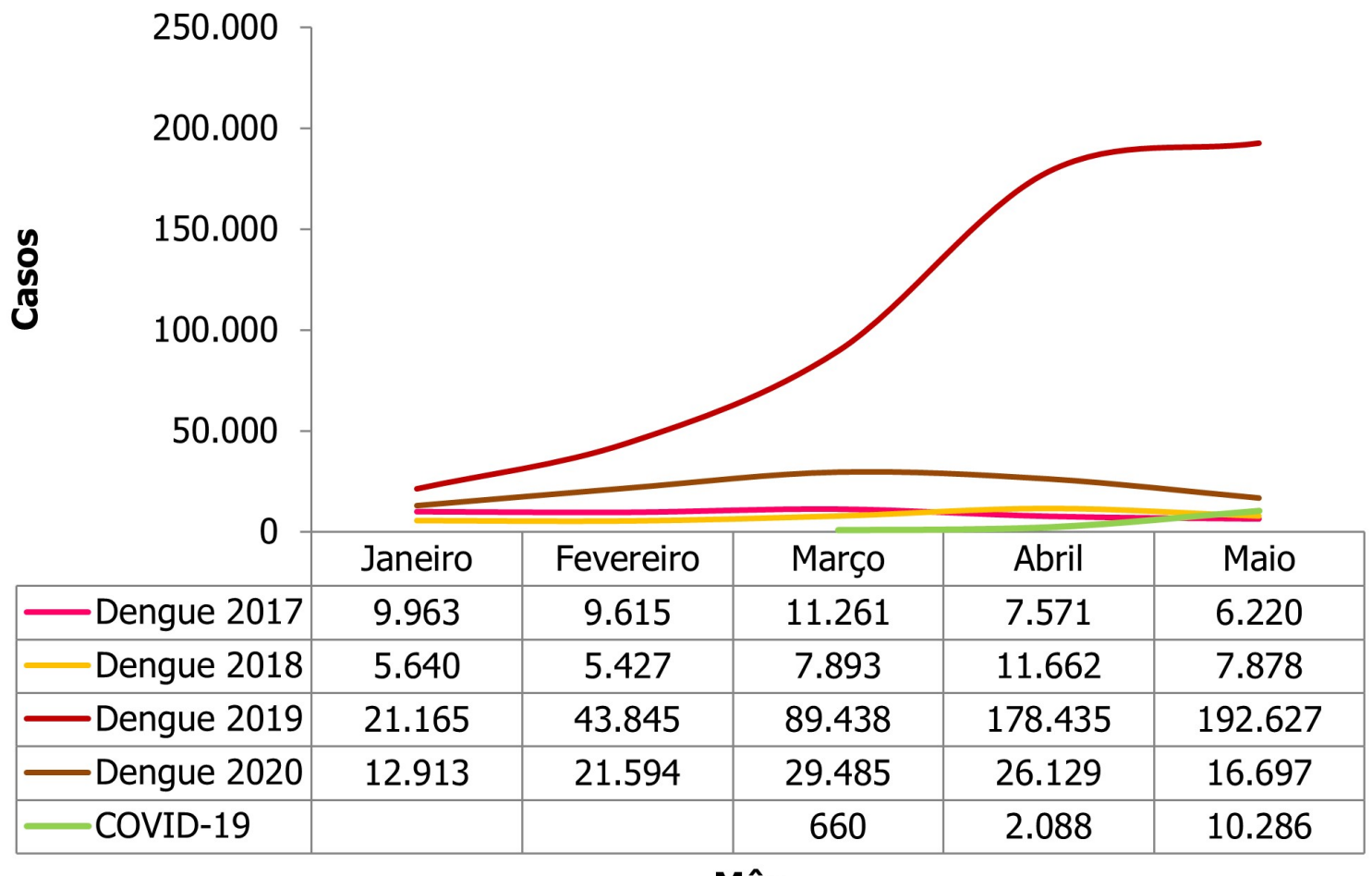

Mês

Fonte: Elaborada pelos autores.

Os locais com maior número de óbitos foram a macrorregião Centro, com 108, seguido do Sudeste, com 60, Sul, com 40, e Triângulo do Norte, com 31 óbitos. Juntos tais locais somavam, até o dia 02 de junho, 74,22\% de todas as mortes por COVID-19 no estado. Ao analisar a velocidade da propagação da infecção no estado de Gerais foi avaliado a variação, em porcentagem, da 20ạ semana epidemiológica sobre a 23ạ semana. As macrorregiões que apresentaram maior elevação no número de casos em 4 semanas foram: Vale do Aço, com $860,8 \%$ de acréscimo, seguido do Noroeste, com $681,2 \%$, Leste, com 590,8\%, Leste do Sul, com 564,3\%, e Centro Sul, com 554,2\%. 
Figura 2 - Número de casos e óbitos por dengue, por mês do ano, no estado de Minas Gerais, de 2017 a 2019.

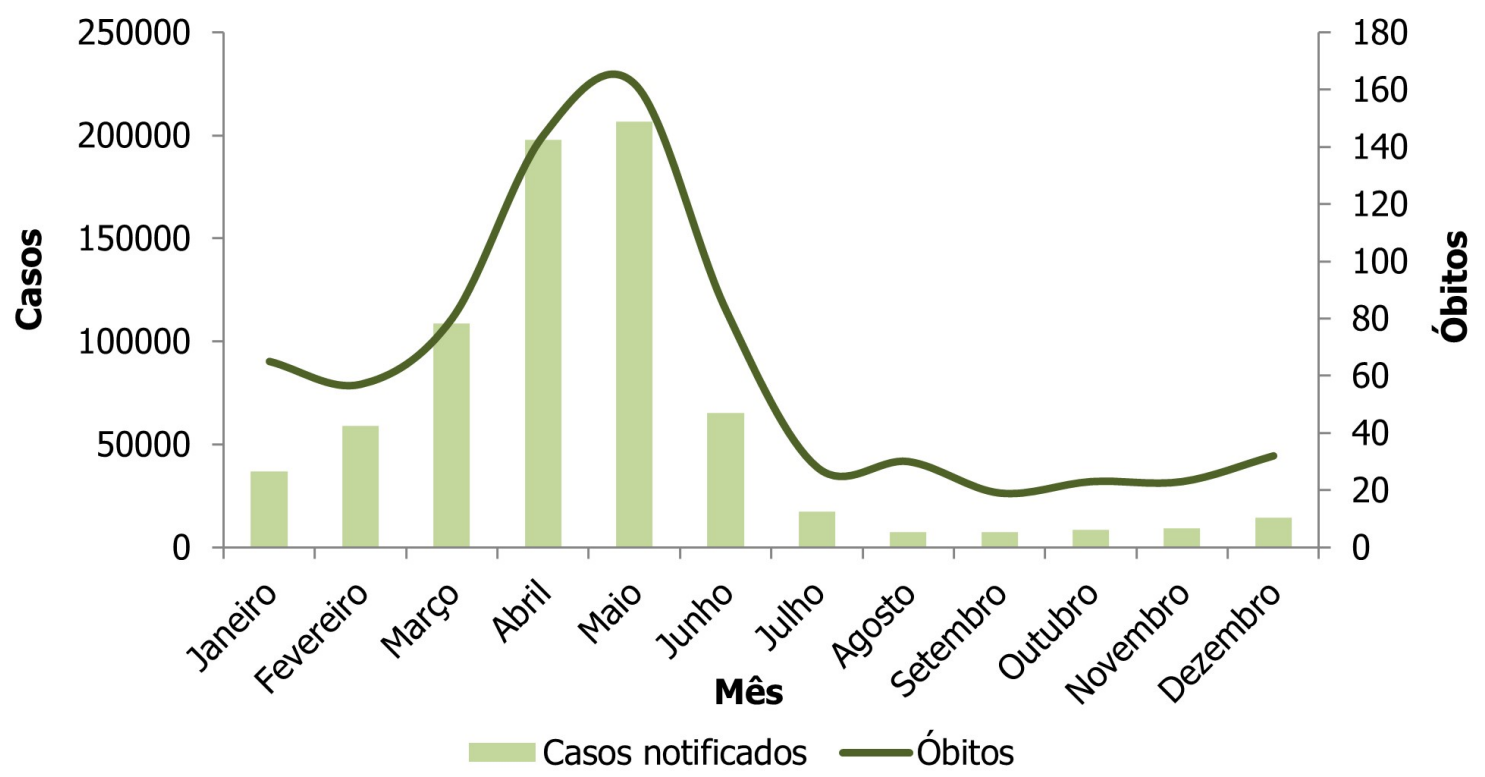

Fonte: Elaborada pelos autores.

Com relação ao número de leitos do SUS, especificamente aqueles que registram internações no SUSFácil, de acordo com o Painel da COVID de Minas Gerais, da SESMG, no dia 12 de junho de 2020 haviam 2.855 leitos de UTI adulto e 12.928 leitos clínicos. Dos leitos de UTI, 2.071 estavam ocupados (taxa de ocupação de 71,60\%), sendo que 327 destes estavam com pacientes com COVID-19 ou com suspeita, significando $11,61 \%$ dos leitos de UTI ocupados. Já os leitos clínicos, 8.770 estavam ocupados (taxa de ocupação de 72,55\%), sendo que 1.039 destes estavam com pacientes com COVID-19 ou com suspeita da doença, significando 8,60\% dos leitos clínicos ocupados.

Observando-se a taxa de ocupação nas macrorregiões, o Triângulo do Norte apresentava a situação mais preocupante até o dia 12 de junho de 2020 , onde $90 \%$ ou mais dos seus leitos de UTI e clínicos estavam ocupados, sendo que daquele 5,26\% são internações pela COVID-19 ou suspeita da doença, e deste $4,87 \%$. Outro local que apresenta alta taxa de ocupação nos leitos clínicos é a macrorregião Centro, sendo que $17,71 \%$ dos ocupados são por COVID-19 ou suspeita, porém, com relação aos leitos de UTI, a taxa de ocupação é de 0 a $40 \%$.

O Vale do Aço, Noroeste, Nordeste, Leste e Jequitinhonha estão com a taxa de ocupação dos leitos de UTI de $90 \%$ ou mais, sendo que por COVID-19 ou suspeita, as taxas são $18,75 \%, 18,97 \%, 26,47 \%, 13,51 \%$ e $4,17 \%$, respectivamente.

De acordo com o Portal da Transparência do Estado de Minas Gerais, no dia 13 de junho foi registrado um gasto com contratos emergenciais firmados para a aquisição de bens, serviços, inclusive de engenharia, obras, alienações e locações para o enfrentamento da COVID-19, com base na Lei Federal $n^{\circ} 13.979 / 2020$ e na Lei Estadual 
$\mathrm{n}^{\circ}$ 23.640/2020, de $\mathrm{R} \$ 165.879 .596,00$. Com tal verba, o estado adquiriu produtos hospitalares tais com gases, reguladores de pressão e máscaras de proteção descartáveis, além de insumos para construção civil, como equipamentos de rede elétrica e telhas, entre outros. Já os gastos referentes ao Programa 26 - Enfrentamento dos efeitos da pandemia da COVID-19, onde inclui serviços gerais, medicamentos e álcool em gel, houve uma despesa de $\mathrm{R} \$ 72.731 .079,00$.

A tabela 2 apresenta o número de casos e óbitos pela COVID-19 nas 14 macrorregiões do estado, além de outras variáveis relacionadas a assistência promovida pela Secretaria de Estado de Saúde de Minas Gerais.

Tabela 2 - Número de casos, óbitos pela COVID-19 e taxa de ocupação de leitos clínicos e de UTI pela COVID-19 e outras doenças nas macrorregiões de Minas Gerais, da 10ạ semana epidemiológica de 2020 até dia 12 de junho de 2020.

\begin{tabular}{|c|c|c|c|c|c|c|}
\hline Macrorregião & $\begin{array}{l}\mathrm{N}^{\circ} \mathrm{de} \\
\text { casos* }\end{array}$ & Óbitos* & $\begin{array}{l}\text { Taxa de } \\
\text { ocupação } \\
\text { de leitos } \\
\text { clínicos } \\
(\%)\end{array}$ & $\begin{array}{l}\text { Leitos Clínicos } \\
\text { disponibilizados** }\end{array}$ & $\begin{array}{l}\text { Taxa de } \\
\text { ocupação } \\
\text { de UTI (\%) }\end{array}$ & $\begin{array}{l}\text { Leitos UTI Adulto } \\
\text { disponibilizados** }\end{array}$ \\
\hline Centro & 4.663 & 108 & 90 ou mais & 3.772 & $0-40$ & 991 \\
\hline Sudeste & 1.508 & 60 & $40-70$ & 766 & $70-90$ & 115 \\
\hline $\begin{array}{l}\text { Triângulo do } \\
\text { Norte }\end{array}$ & 1.268 & 31 & 90 ou mais & 344 & 90 ou mais & 20 \\
\hline Sul & 1.218 & 40 & $40-70$ & 382 & $70-90$ & 38 \\
\hline Vale do Aço & 711 & 10 & $40-70$ & 404 & 90 ou mais & 92 \\
\hline Oeste & 578 & 8 & $40-70$ & 575 & $70-90$ & 18 \\
\hline Noroeste & 539 & 2 & $40-70$ & 226 & 90 ou mais & 63 \\
\hline Nordeste & 490 & 14 & $40-70$ & 869 & 90 ou mais & 240 \\
\hline Centro Sul & 471 & 10 & $0-40$ & 695 & $70-90$ & 138 \\
\hline Leste & 449 & 8 & $40-70$ & 1.608 & 90 ou mais & 339 \\
\hline $\begin{array}{l}\text { Triângulo do } \\
\text { Sul }\end{array}$ & 422 & 13 & SI & 1.786 & SI & 438 \\
\hline Norte & 285 & 9 & $70-90$ & 652 & $40-70$ & 183 \\
\hline Leste do Sul & 186 & 4 & $40-70$ & 511 & $70-90$ & 65 \\
\hline Jequitinhonha & 23 & 0 & $40-70$ & 338 & 90 ou mais & 115 \\
\hline $\begin{array}{l}\text { Sem } \\
\text { informação }\end{array}$ & 220 & 5 & NA & NA & NA & NA \\
\hline Total & 13.034 & 322 & 75,55 & 12.928 & 71,60 & 2.855 \\
\hline
\end{tabular}

$\mathrm{SI}=$ Sem informação;

*Dados do dia 02/06/2020.

**Dados do dia 10/06/2020.

Fonte: Elaborada pelos autores. 


\section{Edição Especial COVID-19}

\section{DISCUSSÃO}

Durante o período de estudo, entre janeiro de 2017 a abril de 2020, foram registradas cerca de 15,4 mil internações por dengue e febre hemorrágica no estado de Minas Gerais e gastos, aproximadamente 6,5 milhões de reais com serviços hospitalares e profissionais. Ainda nessa região, os gastos com os serviços de saúde tendem a aumentar em virtude do contexto pandêmico da COVID-19. De 6 de março a 12 de junho, quase 3 mil pessoas já haviam sido internadas e foram gastos cerca de 166 milhões de reais com a COVID-19 de acordo com Portal da Transparência do Estado de Minas Gerais (2020) publicado no dia 13 de junho, sendo notório as despesas financeiras que essas enfermidades juntas têm provocado ao SUS.

Evidencia-se, que a dengue e os seus fatores multidimensionais não recebem atenção suficiente por parte do sistema de saúde, uma vez que, embora a doença seja considerada no geral de fácil resolução por meio da atenção primária, é notória sua carga econômica onerosa ao SUS, por meio de custos diretos e indiretos, que são intensificados em situações de diagnóstico tardio ou manejo inadequado, tornando muitos casos graves, necessitados do uso dos serviços de média e alta complexidade hospitalar. (TEICH,2017). Somando-se isso ao cenário de ocupação substancial de leitos de UTI provocada pela COVID-19, bem como ao alto investimento financeiro em ações de saúde para seu enfrentamento, fica claro que o ônus ao sistema de saúde tenderá a aumentar no ano de 2020.

Quanto aos casos notificados de dengue na região de Minas Gerais, entre os anos de 2017 a 2019, nota-se que os meses entre janeiro a maio são os mais críticos, com o número de óbitos tendo seu pico em maio. Essa situação revela a incidência sazonal da doença, visto que a temperatura e a pluviosidade afetam na reprodução do vetor e em sua distribuição, de forma que os fatores climáticos do verão chuvoso com aumento da temperatura coincidem com aumento nos casos de dengue na região. (TEIXEIRA; BARRETO; GUERRA, 1999).

Esse cenário, torna-se ainda mais alarmante devido à possibilidade de co-epidemias diante da coincidência temporal de ambas enfermidades, haja vista que os casos provocados pelo novo coronavírus são crescentes desde o início do ano no estado. Como se nota, em apenas uma semana, o cumulativo de casos para COVID-19 cresceu 23,53\% do dia 2 de junho para o dia 9 de junho em Minas Gerais, ocupa até então a 13a posição no "ranking" brasileiro. Ao passo que, para a dengue, segundo o Boletim Epidemiológico número 7 (2020), o estado de Minas Gerais estava em 3o lugar no "ranking" nacional em relação aos casos de dengue.

Observando a situação de Minas Gerais no contexto brasileiro, ao comparar os casos de dengue nos meses de maior incidência, entre os meses de janeiro a maio de 2019 (ano epidêmico) a 2020, tem-se uma redução de quase $80 \%$ e, até o mês de maio de 2020, segundo o gráfico 1, haviam 106.818 casos notificados, enquanto que no mesmo período em 2019 foram notificados 525.510. Nesse viés, tal situação pode estar relacionada, dentre outros fatores, com o atual contexto de pandemia da COVID19 e a subestimação dos efeitos da dengue, de tal modo que a redução do número de 
casos pode sugerir uma possível subnotificação no estado, por esperar-se um número maior em decorrência do período sazonal da doença.

Analisando os leitos de UTI disponíveis em Minas Gerais, nota-se que as macrorregiões de saúde do estado possuem notáveis divergências na capacidade de atender enfermidades que necessitam de assistência hospitalar. É válido destacar que a regionalização do estado divide os municípios mineiros em 89 microrregiões de saúde e 14 macrorregiões de saúde, de modo que a atenção primária deve ser organizada no nível municipal, enquanto que os serviços de média e alta complexidade devem ser direcionados à nível de microrregião e macrorregião respectivamente. (OLIVEIRA; SIMÕES; ANDRADE, 2008). Diante disso, ao considerar a pluralidade assistencial entre as macrorregiões, a pandemia por COVID-19 exige do sistema de saúde desde orientações prestadas pela atenção básica até a demanda de oferta de leitos e equipamentos de ventilação assistida, os quais são de vital importância no tratamento dessa doença em casos graves, afetando o SUS em níveis diferentes de complexidade. (BRASIL, 2020).

Quanto às internações decorrentes da dengue, nota-se de certa forma uma incidência heterogênea entre as macrorregiões analisadas. No período de internação analisado de 2017 a abril de 2020 apenas o Centro manteve-se entre as 3 macrorregiões com o maior quantitativo de internações. Enquanto, sob foco da disponibilidade de leitos em 2020, a taxa de ocupação pela COVID-19 nos leitos de UTI e nos leitos clínicos era de $11,61 \%$ e $8,60 \%$ respectivamente no estado de Minas Gerais, segundo o Painel da COVID do dia 12 de junho. Nesse contexto, 6 macrorregiões já possuíam $90 \%$ ou mais dos seus leitos de UTI ocupados. Assim, percebe-se como é recorrente a ocupação dos leitos de UTI e clínicos.

Sobre outra perspectiva, a dinâmica de transmissão de dengue e da sua incidência envolve variados fatores determinantes, como a questão sócioeconômica da região sob influência da urbanização. (BRASIL, 2010). De maneira semelhante, como a transmissão de SARS-CoV-2 é por contato, esta depende de fatores como aglomeração, que está intimamente ligado à densidade populacional. Por conseguinte, ao considerar que o estado de Minas Gerais possui divergências sociais, geográficas e econômicas, nota-se que as macrorregiões não são igualmente afetadas pelas consequências da dengue e do novo coronavírus.

Assim, das 14 macrorregiões existentes em Minas Gerais, vale destacar o Centro por apresentar o maior número de internações por dengue, sendo quase 5 mil no período analisado, com o maior número de óbitos, 35 dos 158 óbitos registrados no estado, além de onerar cerca de 2 milhões de reais ao sistema de saúde, por meio de gastos com serviços hospitalares e profissionais, o que representa cerca de $33 \%$ dessas despesas do estado somente com a essa doença.

Ainda sobre essa macrorregião, quando se trata da COVID-19, nota-se que a região registrou cerca de 4,7 mil casos, 108 óbitos e taxas preocupantes de ocupação de leitos UTI e clínicos. Vale salientar que possui municípios com alta densidade 
demográfica, como a capital do estado, Belo Horizonte, possuindo 7.167,00 hab/km² (BRASIL, 2010), com 2.144 notificações da COVID-19 (46\% da Macrorregião).

Observando-se a conjuntura acima, nota-se que o Centro, embora seja uma região economicamente favorecida em relação às demais, com o maior número de leitos de UTI e com aparato tecnológico em saúde em centros de média e alta complexidade capazes de controlar doenças mais graves, tal região ainda possui déficit no que tange ao controle da dengue. Cenário que, por sua vez, reforça a falta de investimento em estratégias efetivas na atenção primária para combate de epidemias recorrentes.

Ademais, a macrorregião de saúde Nordeste também se destaca no quesito da ocorrência concomitante de dengue e COVID-19. Essa região ocupa a 60 posição entre as demais no período estudado para o número de internações realizadas por dengue, cerca de mil hospitalizações e com valor médio aproximadamente de $\mathrm{R} \$ 325$ reais gasto por internação. Pela análise dos resultados, nota-se que o Nordeste possui o menor número total de leitos UTI quando se compara com as demais macrorregiões do estado, fator que se torna ainda mais crítico com a presença da COVID-19 em 2020. Até o dia 2 de junho, haviam 490 casos da doença e com ocupação de $90 \%$ dos leitos disponíveis na região.

Esse cenário aponta relativa discrepância entre as macrorregiões do estado de Minas Gerais no que tange à distribuição dos equipamentos de saúde, bem como à eficiência na atuação da atenção primária à saúde. Visto que, pela falta de manejo adequado da dengue, há a necessidade do uso dos serviços prestados pelas unidades de saúde de maior densidade tecnológica, bem como sistemas de apoio, concentrados nos municípios principais das macrorregiões.

Outra macrorregião que vale destacar é a Vale do Aço, que ocupa o 1ㅇ lugar na lista das macrorregiões sobre o valor médio gasto por internação com a dengue, cerca de $\mathrm{R} \$ 500$ reais, demonstrando os gastos direcionados às complicações de saúde oriunda da dengue. Tal macrorregião, ainda que possua menor dimensão territorial, também se destacou em relação a COVID-19, ao subir da 7ạ para a 5a posição, até a semana de referência do boletim epidemiológico número 7 (2020), em 4 semanas foram 860,8\% de acréscimo nos casos. Sendo o município de Ipatinga destaque na macrorregião e um dos responsáveis pelo alto ritmo de crescimento. Observa-se, assim, como o aumento exorbitante do número de casos ocorre em um curto período de tempo e demanda ainda mais do sistema de saúde, já exigido pelos gastos com a dengue.

No âmbito dos cuidados primários, os médicos da atenção primária desempenham um papel crucial tanto no aconselhamento de pacientes com suspeita de dengue para tomar medidas preventivas precoces à cadeia de transmissão dentro da comunidade, quanto no acompanhamento dos casos da COVID-19 para evitar seu agravamento, haja vista que a maioria dos casos não são graves. Dessa forma, torna-se possível desenvolver práticas de educação em saúde visando reduzir a transmissão de ambas enfermidades, considerando os aspectos relacionados à incidência e à distribuição das doenças na sociedade. (TEIXEIRA; BARRETO; GUERRA, 1999) (BRASIL, 2020). 
Nesse sentido, a prevenção da dengue terá um impacto significativo na disponibilidade de leitos, além de diminuir, consideravelmente, o dispêndio do SUS. Tal fato, somado à ampliação da rede hospitalar, ao redirecionamento e à reorganização de serviços, amenizará a situação caótica provocada pela COVID-19.

Por fim, como um dos princípios do SUS refere-se à equidade ao tratar desigualmente os desiguais, para que todas as macrorregiões tenham o mesmo atendimento, é notório o reconhecimento das desigualdades com o objetivo de diminuí-las. Com isso, para que a equidade seja feita deve-se investir na redistribuição dos recursos financeiros. Além disso, salienta-se a importância da prevenção, por não haver vacinas, assim os profissionais da atenção primária devem realizá-la junto à vigilância entomológica da dengue visando a modificação dos comportamentos de risco. Somado a isso, é necessário a cura das pessoas enfermas a fim de reduzir as consequências mais graves da doença mediante a detecção prévia e o tratamento precoce dos casos na rede de atenção primária. (TEIXEIRA; BARRETO; GUERRA, 1999).

Entre os empecilhos do estudo, nota-se a dificuldade de acesso aos dados mais recentes da dengue no SINAN da SVS-MG, bem como na dificuldade de se obter os novos casos da COVID-19, lançado semanalmente de forma cumulativa. Entretanto, o Ministério da Saúde mudou o modelo de divulgação a partir do dia 12 de junho. 0 presente estudo não calculou o Coeficiente Geral de Mortalidade por si só, usando o número de óbitos e expressando por mil habitantes, coletando, apenas, os dados gerados pelo TabWeb do SIH-SUS.

\section{CONCLUSÃO}

Observa-se que foram gastos quase 6,5 milhões de reais, entre 2017 e 2020, com a dengue e 165 milhões de reais disponibilizados para gastos com o novo coronavírus, em 2020, e a macrorregião centro apresenta o maior número de casos para as duas doenças. Além de a região ser uma das mais numerosas e com alta densidade populacional, aliado ao contexto da COVID-19 possibilidade de sobrecarga do SUS é alta, tendo em vista que os meses de abril, maio e junho caracterizam o período mais crítico para a dengue. Assim, são necessários investimentos, considerando as vulnerabilidades macrorregionais que assegurem a tomada de medidas preventiva.

\section{REFERÊNCIAS}

BRASIL. Protocolo de Manejo Clínico do Coronavírus (COVID-19) na Atenção Primária à Saúde - Versão 9. Brasília: Ministério da Saúde, Secretaria de Atenção Primária à Saúde, mai 2020. Disponível em: https://www.saude.gov.br/images/pdf/ 2020/marco/20/20200318-ProtocoloManejo-ver002.pdf. Acesso em: 8 jun. 2020.

BRASIL. Sistema de Informações Hospitalares do SUS: Morbidade Hospitalar do SUS (SIH/SUS). Brasília: Ministério da Saúde, 2020. Disponível em: http://www2. datasus.gov.br. Acesso em: 10 de jun. 2020. 
BUSS, P. M.; PELLEGRINI, F. A. A saúde e seus determinantes sociais. Physis, v.17, n.1, p.77-93, 2007.

IBGE. Área territorial brasileira. Rio de janeiro: Instituto Brasileiro de Geografia e Estatística, 2018.

IBGE. Censo Demográfico 2010: área territorial brasileira. Rio de Janeiro: Instituto Brasileiro de Geografia e Estatística, 2011.

IBGE. Censo Demográfico. Rio de Janeiro: Instituto Brasileiro de Geografia e Estatística, 2010.

IBGE. Cidades e Estados. Rio de Janeiro: Instituto Brasileiro de Geografia e Estatística, 2018.

IBGE. Estimativa da População. Rio de Janeiro: Instituto Brasileiro de Geografia e Estatística, 2019.

LORENZ, C.; AZEVEDO, T. S.; CHIARAVALLOTI-NETO, F. COVID-19 and dengue fever: a dangerous combination for the health system in Brazil. Travelmedicine andinfectiousdisease, v.35, 2020.

MINAS GERAIS. Tabulador de informações de saúde (TABNET). Belo Horizonte: Portal de Vigilância em Saúde, 2020. Disponível em: http://vigilancia.saude.mg.gov.br/ index.php/informacoes-de-saude/informacoes-de-saude-tabnet-mg/. Acesso em: 10 jun. 2020.

MINAS GERAIS. Boletim Epidemiológico COVID-19: Doença causada pelo coronavírus - 12 de junho de 2020. Belo Horizonte: Secretaria de Estado de Saúde, 2020. Disponível em: https://www.saude.mg.gov.br/images/noticias_e eventos/ 000_2020/Boletins_Corona/Boletim_Epidemiologico_COVID-19_12.06.2020.pdf. Acesso em: 20 jun. 2020.

MINAS GERAIS. Boletim Epidemiológico e Assistencial COVID-19 (Edição

Especial) Número 5. Belo Horizonte: Secretaria de Estado de Saúde, 2020.

Disponível em: http://coronavirus.saude.mg.gov.br/boletim. Acesso em: 01 jun. 2020.

MINAS GERAIS. Boletim Epidemiológico e Assistencial COVID-19 (Edição

Especial) Número 7. Belo Horizonte: Secretaria de Estado de Saúde, 2020.

Disponível em: http://coronavirus.saude.mg.gov.br/boletim. Acesso em: 12 jun. 2020.

MINAS GERAIS. Deliberação CIB/SUS-MG no 3.138, 26 de março de 2020. Aprova a distribuição de recursos do Bloco de Custeio das Ações e Serviços Públicos de Saúde, previsto na portaria GM/MS no 480, de 23 de março de 2020 , destinados às ações de saúde para o enfrentamento do Coronavírus - COVID 19 no estado de Minas Gerais. Belo Horizonte: Secretaria de Estado de Saúde, 2020. Disponível em: https://www.saude.mg.gov.br/images/documentos/Del\%203138\%20-\%20Recusos \%20Portaria\%20480.pdf. Acesso em 19 jun. 2020. 
MINAS GERAIS. Deliberação CIB-SUS/MG no 3.013, de 23 de outubro de 2019. Aprova o Ajuste/2019 do Plano diretor de Regionalização PDR/SUS MG e dá outras providências. Belo Horizonte: Secretaria de Estado de Saúde, 2020. Disponível em: https://www.saude.mg.gov.br/images/documentos/Del\%203013\%20-\%20SUBGR SDCAR_DREA\%20-\%20Ajuste\%20PDR\%20vers\%C3\%A30\%20CIB\%20-\%20alterada \%2015.10.pdf. Acesso em: 20 jun. 2020.

MINAS GERAIS. Painel Coronavírus. Belo Horizonte: Secretaria de Estado de Saúde, 2020. Disponível em: http://coronavirus.saude.mg.gov.br/painel. Acesso em: 10-12 jun. 2020.

MINAS GERAIS. Portal da Transparência do Estado de Minas Gerais. Belo Horizonte: Secretaria de Estado de Saúde, 2020. Disponível em: http://www. transparencia.mg.gov.br/covid-19. Acesso em: 13 jun. 2020.

MINAS GERAIS. Resolução SES № 7045, de 3 de março de 2020. Divulga oS valores e beneficiários do incentivo financeiro complementar para os municípios com alta incidência de Dengue, Chikungunya e Zika, nos termos da Deliberação CIB/SUSMG n³.126/2020. Belo Horizonte: Secretaria de Estado de Saúde, 2020. Disponível em:https://www.saude.mg.gov.br/images/documentos/RESOLU\%C3\%87\%C3\%830 \%207045\%20PDF\%20SITE.pdf. Acesso em: 20 jun. 2020.

MINISTÉRIO DA SAÚDE. Resolução no 466, de 12 de dezembro de 2012. Diretrizes e normas regulamentadoras de pesquisa envolvendo seres humanos. Brasília: Diário Oficial da União, 2012. Disponível em: https://bvsms.saude.gov.br/bvs/saudelegis/cns/ 2013/res0466_12_12_2012.html. Acesso em: 5 mai. 2020.

MINISTÉRIO DA SAÚDE. Boletim Epidemiológico 23. Monitoramento dos casos de arboviroses urbanas transmitidas pelo Aedes (dengue, chikungunya e Zika), Semanas Epidemiológicas 1 a 22, 2020. Brasília: Secretaria de Vigilância em Saúde, 2020.

Disponível em: https://www.saude.gov.br/images/pdf/2020//une/09/Boletimepidemiologico-SVS-23.pdf. Acesso em: 29 jun. 2020.

OLIVEIRA, A.C.; SIMÕES, R.F.; ANDRADE M.V. Regionalização dos serviços de média e alta complexidade hospitalar e ambulatorial em Minas Gerais: estrutura corrente versus estrutura planejada. In: SEMINÁRIO SOBRE ECONOMIA MINEIRA, 13., 2008, Diamantina. Anais... Diamantina: UFMG/Cedeplar, 2008.

OMS. Discurso de abertura do Diretor-Geral da OMS no briefing da mídia sobre COVID-19 - 11 de março de 2020. Genebra: Organização Mundial da Saúde, 2020. Disponível em: https://www.who.int/dg/speeches/detail/who-director-general-sopening-remarks-at-the-media-briefing-on-covid-19---11-march-2020. Acesso em: 15 jun. 2020.

TEICH, V.; ARINELLI, R.; FAHHAM, L. Aedes aegypti e sociedade: o impacto econômico das arboviroses no Brasil. JBES: BrazilianJournalof Health Economics/Jornal Brasileiro de Economia da Saúde, v.9, n.3, 2017. 
TEIXEIRA, M. G.; BARRETO, M. L.; GUERRA, Z. Epidemiologia e medidas de prevenção do Dengue. Informe Epidemiológico do SUS, v.8, n.4, p.5-33, dez. 1999.

YAN, G. et al. Covert COVID-19 and false-positive dengue serology in Singapore.

Lancet InfectDis, v.20, n.5, p.536, 2020. Disponível em: https://www.thelancet.com/ pdfs/journals/laninf/PIIS1473-3099(20)30158-4.pdf. Acesso em 13 jun. 2020. 